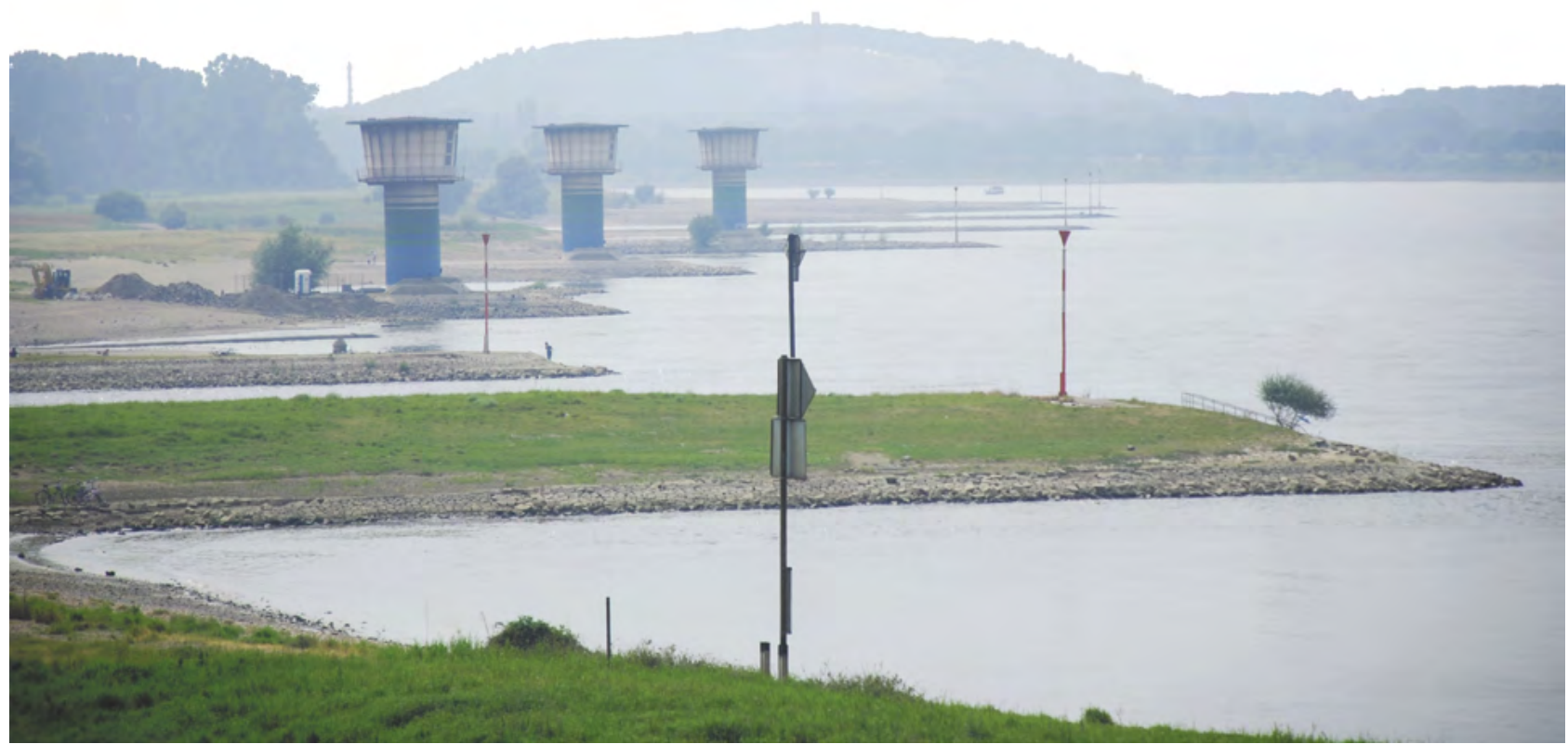

Faculté d'architecture, d'ingénierie architecturale, d'urbanisme de l'Université catholique de Louvain 


\title{
TVBUONAIR au CREAT
}

Trame verte et bleue en milieu urbanisé

\author{
Yves Hanin (dir.), Alexandre Leclercq, Fiorella Quadu, \\ Martin Grandjean, Ségolène Gréant,
}

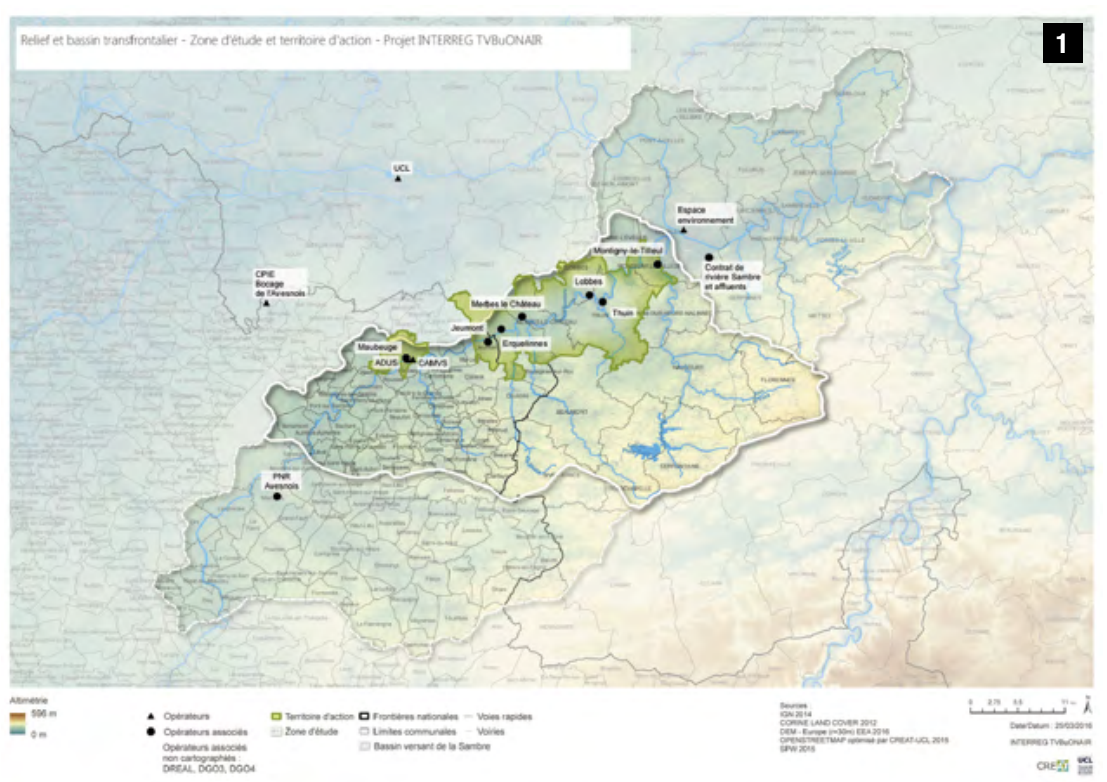

\section{Une recherche-action transfrontalière}

La recherche TVBuONAIR (Trame verte et bleue en milieu urbain en direct) consiste à répondre aux enjeux territoriaux de remise en état des corridors écologiques de part et d'autre de la frontière franco-belge sur le bassin de la Sambre. Elle vise spécifiquement les milieux urbanisés.

Pilotée par le CREAT/UCL, cette recherche-action trouve son originalité dans le type d'acteurs territoriaux qu'elle mobilise de chaque côté de la frontière, acteurs évoluant dans des sphères institutionnelles et avec des marges de manœuvre variées. Elle est financée en partie par le programme européen Interreg et par la Région wallonne.

TVBuONAIR s'inscrit dans une démarche d'aide à la décision visant la reconnexion des habitats naturels en milieu urbanisé, la lutte contre la fragmentation des habitats par la construction de projets de cohérence écologique et plus globalement la réintroduction de la nature en ville dans le bassin nord de la Sambre.

Au-delà de l'enjeu lié au maintien et à la restauration de la biodiversité en milieu urbain, il s'agit aussi d'inscrire cette recherche-action dans une ambition de requalification de l'image territoriale de cette région fortement liée à son passé sidérurgique.
La recherche-action aborde ainsi la question de la réintroduction de la nature en ville :

- en mutualisant les connaissances des différents acteurs du territoire, du patrimoine et de l'environnement ;

- en créant des outils de gestion et de planification intégrées à l'échelle d'un bassin versant transfrontalier ;

- en sensibilisant la population, les techniciens et les élus ;

- et enfin, par la réalisation d'actions locales à des échelles variées visant à favoriser la biodiversité urbaine.

\section{Un partenariat}

\section{multidisciplinaire, une}

\section{recherche transdisciplinaire}

Le partenariat multidisciplinaire de TVBuONAIR apporte une expertise et une complémentarité opérationnelles au sein du territoire et vise ainsi une approche interdisciplinaire forte. En effet la recherche nécessite une approche transversale mettant en relation différents champs de compétences comme l'évaluation prospective des besoins d'urbanisation par les caractéristiques socio-économiques des populations ; la maîtrise des outils de gestion territoriale aux échelles régionales et locales et leur mise en œuvre ; la connaissance des politiques connexes (mobilité, logement, patrimoine...) ; l'expérience en matière 


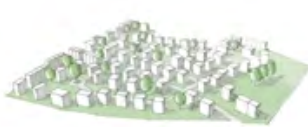

Espace vert urbain avec un habitat dispersé : Habitat individuel

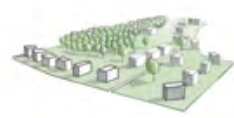

Espace vert urbain avec une faible densité d'habitat

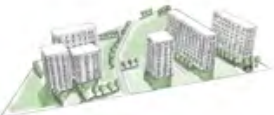

Espace vert urbain avec un habitat dispersé : Habitat collectif

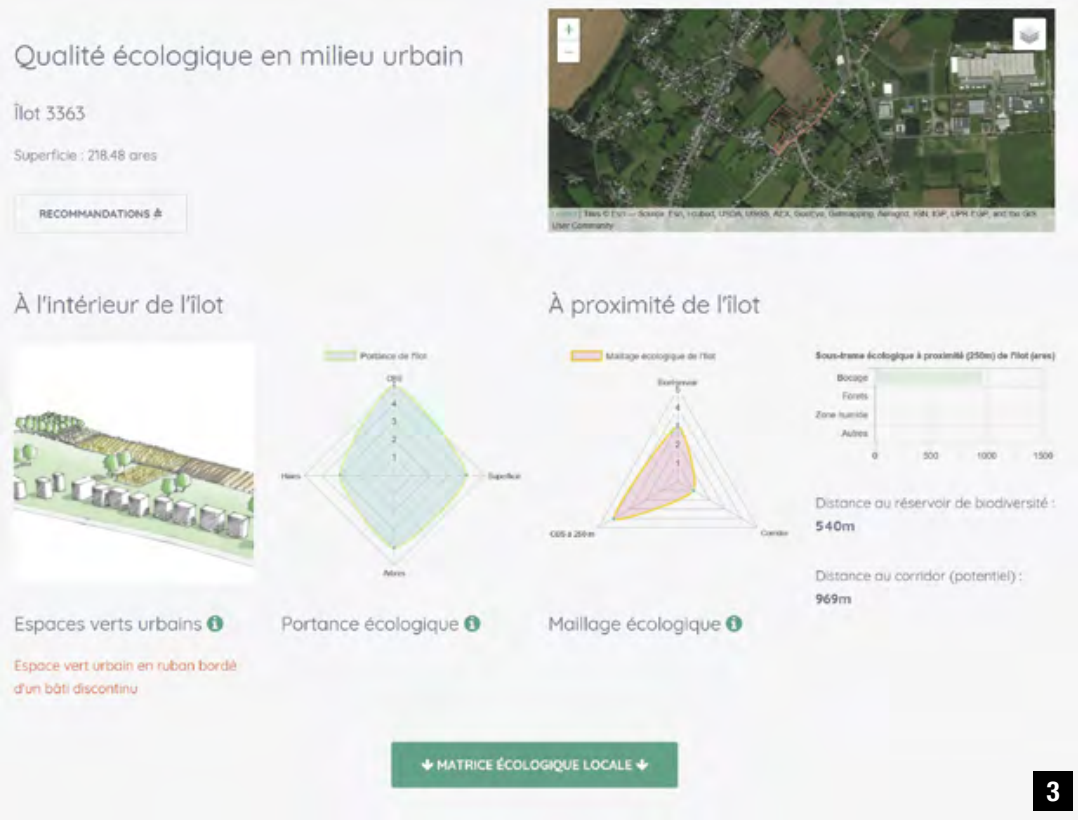

de management public, de communication, de participation ; la pratique du traitement des données et des systèmes d'information géographique (SIG opérationnel).

Parmi les travaux de recherches menés par le CREAT, de nouvelles méthodes de caractérisation des espaces verts urbains privés sont développées et se basent sur la morphologie/structure de ces espaces au sein des tissus urbanisés.

\section{Trois angles d'approches}

La recherche-action TVBuONAIR aborde la nature en ville sous des angles d'analyse non seulement écologique mais également urbanistique et sociologique.

Les approches sociologique et urbanistique prévues au sein des différents volets méthodologiques doivent permettre de lever le clivage ville/nature et de mieux intégrer les besoins des habitants. Cette recherche action s'inscrit donc pleinement dans une approche systémique liée au développement durable des territoires. II s'agit d'aboutir à une réelle cohérence transfrontalière de la trame verte et bleue urbaine par la mobilisation générale de tous les acteurs en croisant trois approches territoriales environnementale, urbanistique et sociologique.
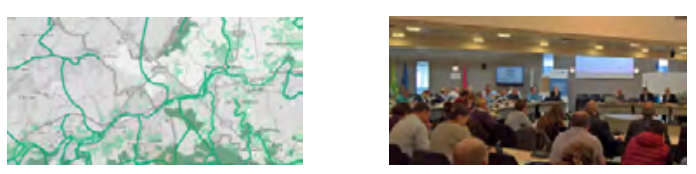

Cohérence transfrontalière à l'échelle du bassin de la Sambre
Dynamique participative et de co-construction

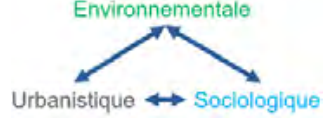

Croisement de différentes approches territoriales
2 Caractérisation des espaces verts urbains privés - exemples. Sources: TVBuONAIR 2017 CREAT-UCL 2017 / Ségolène Gréant 2017

3 L'outil dynamique (Réalisation CREAT, 2017)

4 Les démarches mises en oeuvree TVBuONAIR (Source : CREAT) 
AgGeri, G., 2010, "Inventer les villes-natures de demain : gestion différenciée, gestion durable des espaces verts", Educagri, Paris. CERTU, 2009, "Composer avec la nature en ville" Lyon, Centre d'Etudes sur les Réseaux, les Transports, I'Urbanisme et les Constructions Publiques. Clergeau, Ph., Blanc, N. 2015. Trames Vertes Urbaines. De la recherche scientifique au projet urbain. Le Moniteur. Consalėe J.-N., Goiffon M. et BARTHÉLÉMY C.,"Entre aménagement du paysage et ménagement de la nature à Marseille: la trame verte à l'épreuve du local.", Développement durable et territoires [En ligne], Vol. $3, n^{\circ} 2$ |Juillet 2012, consulté le 6 août 2017. URL : http://journals. openedition.org/developpementdurable/9268; DOI : 10.4000/developpementdurable. 9268 MeHDI, L., 2010, "Structure verte, Structure verte et biodiversité urbaine". L'espace vert : analyse d'un écosystème anthropisé. Thèse de doctorat, Aménagement, Université de Tours.

MeHdi, Lotfi, etal. "Évolution de la place du végétal dans la ville, de l'espace vert à la trame verte." [VertigO] La revue électronique en sciences de l'environnement, vol. 12 , $n^{\circ} 2$, september 2012 Glatron S., Grésillon E. et Blanc N.,"Les trames vertes pour les citadins : une appropriation contrastée à Marseille, Paris, Strasbourg", Développement durable et territoires [En ligne], vol. 3, n² 2 Juillet 2012, consultéle 10 septembre 2017.URL: http://journals.openedition.org/developpementdurable/9297; DOI : 10.4000/ developpementdurable. 9297 Arrif T., Blanc N. et ClerGEAU P.,"Trame verte urbaine, un rapport Nature - Urbain entre géographie et écologie","Cybergeo European Journal of Geography [En ligne], Environnement, Nature, Paysage, document 574 , consulté le 10 septembre 2017.URL

http://journals.openedition.org/ cybergeo/24862; DOI : 10.4000 / cybergeo. 24862

5 Sensibilisation des élus et des techniciens communaux à la gestion des cimetières nature (Source: CREAT, 2017)

6 Enquête sociologique des usages d'un espace vert à Maubeuge (Source : CREAT, 2017)

\section{Des résultats attendus pour 2020}

Les résultats attendus de TVBuONAIR à son terme en juin 2020 sont le renforcement des échanges transfrontaliers sur l'évaluation, la conservation et la gestion des habitats en milieu urbanisé ; la compréhension des dynamiques de changements des habitats naturels en milieu urbain et l'amélioration de la connaissance des milieux naturels transfrontaliers ; le développement d'une méthodologie commune, durable et transfrontalière pour évaluer la fragmentation des habitats et prendre en compte ces données dans les programmes d'aménagement ; la mise en réseau des acteurs et l'accès à l'information du patrimoine naturel transfrontalier des utilisateurs concernés, autorités de planification, propriétaires, promoteurs, etc; l'évolution des mécanismes de prise de décision à intégrer davantage la biodiversité et les habitats naturels lors de projets d'aménagement du territoire ; la sensibilisation des publics très variés à l'importance de l'environnement naturel en milieu urbain ; la création de projets communs de valorisation à des échelles variées (la rue, l'ilot, le quartier, la ville...).

\section{Une recherche-action qui s'inscrit dans les travaux du CREAT}

L'innovation de cette recherche-action réside dans son approche transfrontalière et multiscalaire de la trame verte et bleue en milieu urbanisé. Elle s'inscrit dans deux des principaux axes d'études du CREAT : le développement territorial et la composition territoriale.

Le premier se base sur l'évaluation interdisciplinaire, participative et prospective permettant de capitaliser et d'approfondir les connaissances du territoire. Le champ de recherches investigué est l'espace comme capital de ressources à préserver, à valoriser et à renouveler.

Le second trouve sa finalité dans la réhabilitation de sites à enjeux environnementaux d'une échelle locale à une échelle transfrontalière suprarégionale. On y développe des lieux et des paysages renforçant la structure écologique.

Plus d'informations :

https://www.tvbuonair.eul http://www.creat-uclouvain.be

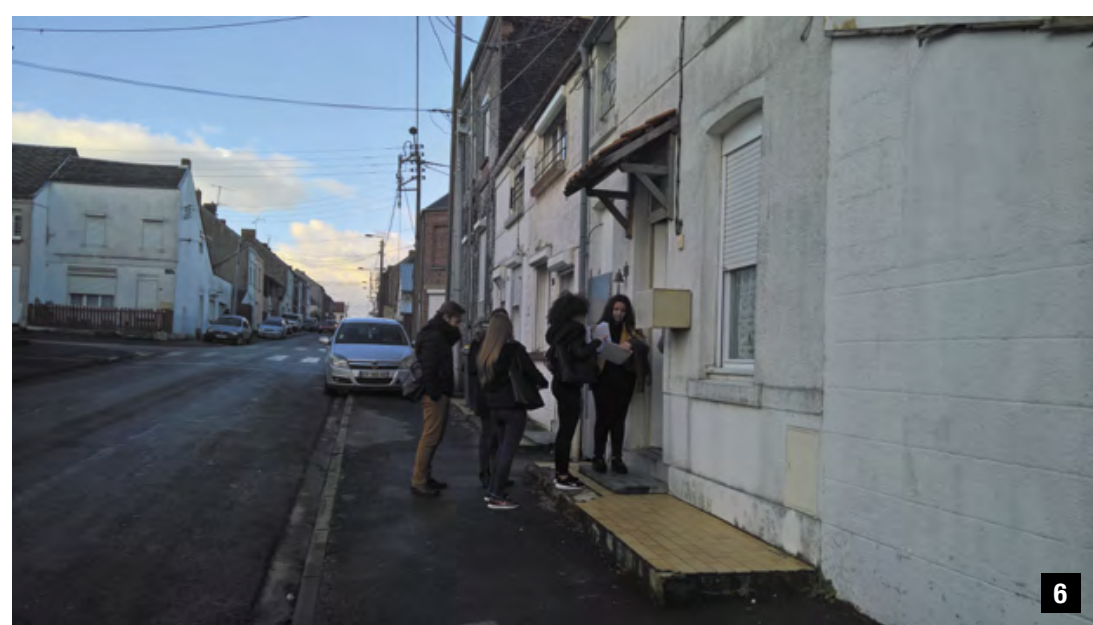

